\title{
Quality in Dental Practice: A Review
}

\section{NISHANT MEHTA', ASHA WADHWA², NITIKA CHAWLA ${ }^{3}$}

The Indian healthcare industry is experiencing rapid transformation owing to the ever-rising demand for quality healthcare. With the increased standard of living in India people are becoming health conscious, shaping a new market which is giving increasing importance to healthy teeth and dental cosmetics. Though great deals of funds have been raised and numerous acts and institutions formulated to up bring the health status of poor and needy ones and ensure equitable distribution of dental health care facilities. To deliver the dental services to masses and improve the quality in dental practices, standards have been set which will ensure that masses receive high quality of dental care and treatment. Various modes to measure the quality in dental care have been implemented but still certain challenges act as hindrance to deliver proper quality of dental practice. Despite of that improvement has taken place in the field of dental practice. Still further improvement can be done by increasing manpower, its equal distribution, raising funds to improve the quality, opening new government colleges and timely inspection of existing educational institution. Properly synchronized strategies need to be developed for improving quality in dental practice in India harbouring all the needs of the consumers and dental personnel.

\section{KEYWORDS: Quality, Dental practice, Dental care}

\section{INTRODUCTION}

The Indian healthcare industry is experiencing rapid transformation owing to the increased demand for quality healthcare. With the increased standard of living in India people are becoming health conscious, shaping a new market which is giving increasing importance to healthy teeth and dental cosmetics. The potential size of India's dental market is huge and as per Indian Dental Association, India is lined up to become one of the largest importer of dental products and materials. As of date, the Indian dental care services market is anticipated at approximately US\$ 600 million and dental equipment and appliances market is about US\$ 90 million, with a 10\% yearly growth rate. As per Cygnus Business Consulting and Research 2010 report, the Indian dental equipments industry is expected to reach US\$ 116.43 million, the dental care services market to US\$ 1.16 billion and oral health care market to US $\$ 1.8$ billion by the year 2014. ${ }^{1}$

Unfortunately this is only side of the coin representing the flourishing Indian dental market and the other side represents the still grave rural and less privileged populations' front. Though great deals of funds have been raised and numerous acts and institutions formulated to up bring the health status of poor and needy ones and ensure equitable distribution of dental health care facilities. Moreover, the lack of tools to measure the quality of dental practice across the country place an additional hindrance to assess the value and volume of effective care delivered surpassing all inequalities of region and financial barriers.

\section{STANDARDS OF DENTAL CARE AND TREATMENT}

Dental services are delivered to masses at various levels in various modes: Primary Care private dental services, including private treatments provided alongside Health Service treatments in mixed practices; and Primary Care Health Service dental services, including those provided by the Community Dental Service. Primary Care relates to the local dental services available directly to patients at their door steps, such as those services from High Street dentists and Oral Health Care Centers. The term Secondary Care refers to hospital or specialist care, to which a patient may be referred from Primary Care. A standard of care has been set to achieve six important aims for quality improvement: ${ }^{2}$
1. Safe: that avoids injuries to patients from the care that is intended to help them.
2. Effective: that provides services based on rationale knowledge to everyone who could benefit and restricts from providing services to those who are unlikely to benefit (avoiding under-use and 
over-use, respectively).

3. Patient-centered: providing care which is deferential of and responsive to individual patient preferences, needs, and values and ensuring that patient values guide all relevant clinical decisions.

4. Timely: which reduces waits and harmful delays for both those who receive and those who give care? 5. Efficient: that avoids wastage, including wastage of equipments, consumables, ideas and/or energy.

6. Equitable: providing care which does not differ in quality in response to personal characteristics like geographic location, socioeconomic status, gender and ethnicity.

These standards are to be adopted by all dentists in a Primary Care, whether Health Service or private setting. Their purpose is to ensure that masses receive consistently high quality care and treatment. The standards can be used to help consumers decide which dental service to choose.

The Quality Standards for Health and Social Care has provided following key quality themes: ${ }^{3}$

1. Accessible, flexible and responsive services: to choose dental care service, before appointment and visits.

2. Efficient, safe and effective care: to assess needs of individual patients, the provision for quality care and treatment, medical emergencies, infection control and environmental care.

3. Effective communication and information

4. Promoting, protecting and improving health and social well being: Ongoing care and attending to special needs of Children, young people and vulnerable adults.

5. Effective communication and information: freedom to express and discuss the treatment options and confidentiality.

6. Corporate leadership and accountability among the dental personnel.

\section{MODES TO MEASURE QUALITY OF DENTAL CARE}

With the rise in national expenditures on healthcare, need to correctly assess quality and efficiency of care has become more vital. Researches have documented variations treatment standards and protocol across different care settings, geographic regions and within treatment providers. Measuring healthcare quality and utilizing the measurements to promote improvements in delivering oral health care have been an agenda of concern for health care administrators and advocates of health.

Various governmental agencies and health organizations are currently involved in the developing and implementing oral health reform measures ${ }^{4}$ of note are:

- Centers for Disease Control and Prevention

- Centers for Medicare and Medicaid Services

- Maternal and Child Health Bureau

- National Committee on Quality Assurance

- Agency for Healthcare Research and Quality

- Health Resources \& Services Administration

- Veterans Administration

- Indian Health Service

- National Network for Oral Health Access

\section{Challenges faced in measuring outcomes in dentistry}

There are substantial amount of challenges to develop and implement measurement guidelines in dentistry. These include:

- Limited knowledge of outcomes

- Limited accessibility of claims data

- Limited diagnostic data collection to establish oral health benchmarks

- Few evidence-based guidelines

- Limited information systems for capturing and transmitting data from patient records

Interviews and questionnaires have been utilized as a common way of collecting information regarding quality of care delivered. ${ }^{5}$ Despite the recognized need to adopt evidence based principles in the delivering oral health care, often this is of limited value due to insufficiencies or inconclusive nature. There is a limited number of high-quality prospective clinical trials on oral health topics, also of concern is the lack of understanding true oral health outcome picture, as dentistry does not have a culture of officially reporting definite diagnoses or relating diagnoses with associated services, especially using claims process. In due course, dentistry will need a cost-effective measurement system to evolve, which could be easily applied on daily basis in small practices, yield significant information that can be acted upon for advancement and could even quantify and record factors under practitioner control. This need to effectively measure and monitor health care 
outcomes and to assure that the public receives optimal benefits from available knowledge and effective care is the basic responsibility of any health care establishment at smaller or national levels. Rapid rise in costs and reported inconsistencies in quality of medical care have culminated in the national priority to deliberately seek valuation of healthcare. Oral health care delivery system should be able to measure what works and what doesn't and make changes needed to improve health outcomes to assure that it is providing the highest quality patient centred dental care.

\section{IMPROVEMENTS IN DENTAL PRACTICE SO FAR PARADIGM SHIFT ROM EXTRACTION TO PREVENTION}

Patients perceived this difference when structured preventive care was introduced. This holds true for patients at every level of risk of developing dental diseases, that is, with healthy mouths and those with presence of risk factors and early disease signs at all set of practices. History of poor oral health is progressing to worsening of oral health without provision for preventive, promotive and economical curative oral health care. ${ }^{6}$ People live with pain as were unable to pay for oral health care treatment and, or are unhappy in this scenario and feel unable to cope up with it as they have to continuously pay for restorative and rehabilitative work.

\section{UPSURGE IN NUMBER OF DENTAL COLLEGES}

With the starting of Dr. R. Ahmed Dental College and Hospital, the first autonomous dental college in Southeast Asia at Calcutta in 1920, the much needed otherwise neglected dental education and dental hospitals which started in India as early as 1883 also gained some direction and momentum. Since then the number of dental schools has significantly increased and gained significant upsurge in the past two decades. A new Comptroller and Auditor General [CAG] report highlighted presence of 292 colleges in India, most of them in private sector and concentrated across only five states. From the year 1960 to 2000 number of graduating dental students has also significantly increased from 1370 to over 260oo; the number is increasing now as well in log rhythmic rates. ${ }^{7}$

\section{AWARENESS AMONGST MASSES}

Patients have now transitioned from their initial stages of being fixed in situations of having decaying and deteriorated teeth to a state where they had gained changes in lifestyle and experienced embellished positive outcomes. Patients have added up new knowledge, developed new clinical relationships and established healthy attitude and practices. Patients value the dental care without much drilling and filling" of teeth and have now started characterizing dentists as either "old-school" or "new-school" based on the treatment options provided to them and the clinical relationship offered. Literature also suggests that patients' perceptions of the quality of dental care and the possibility of them looking for care are related to the acquity of their dentists as caregivers. Many studies describe perceived characteristics of dentists which likely increase care-seeking behaviour or satisfaction of patients to provided care; which include communication skills, informing patients about treatment options, and dental teams' behavior during dental visits. ${ }^{6,8}$

\section{DENTAL CARE STATUS IN RURAL AREAS}

Quality of dental health care provided and its accessibility is determined by many interacting social and environmental factors. Access to oral health care is a more immediate concern for rural residents although delivery of quality care is also important at the same time. Though many major steps have been taken by Indian dental association and impact of mobile dental services has been evident in improving rural health care services. But still the results are not remarkable or commendable. Reports indicate that five states of the country house more than $50 \%$ of the dental schools. Of utmost concern is the geographical imbalance in the dentist to population ratio in India, which stands at around 1:10,00o in urban areas and 1:250,000 in rural areas, which clearly indicates a skewed distribution of oral health care delivery system 9. When there is no accessibility to oral health care in rural India, question of quality of care becomes immaterial. Poverty, tobacco use, transportation barriers and lower access to fluoridated products contribute to greater levels of oral diseases viz. untreated dental decay, permanent loss of teeth and engage in 
smoking and tobacco use, increasing the likelihood of oral cancers, periodontal disease and dental caries but unfortunately the volume of care being delivered is still meager. ${ }^{10}$

\section{HINDRANCES TO QUALITY DENTAL PRATICE ${ }^{11}$}

The fore most challenge for the dental health care delivery system in India is to look after the oral health for a 1.35 billion population is with a very limited budget being sanctioned to health which in turn becomes very meagre for oral health.

\section{FALLING EDUCATION STANDARDS}

There seems to be lack of continuity between planning and implementation. The solution to this could be: ${ }^{10}$

- Carrying out continuing dental education programs to encourage students to prepare ideal as well as provisioning in the dental education protocol to include alternative and related treatment plans thereby improving logical reasoning and analytical skills of new dentists.

- To improve knowledge, skills and attitude of the students and also prepare them for self-directed life-long $\backslash$ learning i.e. creating an environment conducive for problem based $\backslash$ learning [PBL] and evidence based learning.

- Periodic updatation of dental curriculum with emphasis on preventive, promotive and comprehensive oral health care and to facilitate long term self-evaluation by dental students.

\section{LACK OF RESEARCH IN DENTAL SET-UP}

Under graduate level dental programs in India has very little scope for research activity. The reasons range from lack of funds to lack of research mentors. The BDS curriculum also lacks formal training in research methodology. Moreover, the problem being faced in research centre is the absence of original research and duplicacy of results leading to pooling up of fake data.

\section{INCOMPETENT FACULTY}

Recent reports indicate that shortage of teaching faculties in some dental colleges ranged from 8-97 percent. Most of the faculties lack diligent research training and are ill equipped to guide students into research in spite of having completed a postgraduate program [MDS]. Similarly, very few dental colleges have the facility for staff evaluation, by the students, and in those few colleges where such system exists there is little control over biased opinions.

\section{PRESERVING ETHICS IN DENTAL PRACTICE}

Unethical practices are common in spite of the existence of a code of Dental ethics. Hardly any monitoring and guiding mechanism exist to prevent deviation from normal ethical practices. Lack of awareness among dentists and will coupled with an inefficient official mechanism capable of enforcing ethical practice might be the responsible factors. Furthermore, undergraduate curriculum lacks formal training on medical and dental ethics.

\section{LACK OF EMPLOYMENTS AND FINANCIAL SECURITY AMONGST PRACTITIONERS}

Dental colleges are mushrooming like never before, and proportionally the number of under graduate passing out from these colleges is rising in great proportions, but there seems to be lack of due concern and serious thinking regarding the future and profitable employability of a dental graduate. The ground reality is that the pay package of a senior lecturer or young faculty in a private dental college in most of the cities and even the rural towns of the country is in fact less than those of skilled labourers.

\section{LACK OF GOVERNMENT FUNDED DENTAL AIDS AND RECOMMENDED DENTAL HEALTH INSURANCES}

The strategies employed by government to improve oral health status fail to take upon a wholesome approach and target each and every individual irrespective of age, gender and socio economic status. The oral diseases are rampant and affect every sector of people due to lenience at the part of government practitioners, lack of funds and perseverance, the deliverance of health care is inefficient. The government and private sectors still don't consider the oral heath under the health insurance schemes, thus making it an unattended sector.

\section{SKEWED MANPOWER DISTRIBUTION}

The manpower in dental care sector in India is near to adequate but the problem lies in its skewed distribution. There are many states viz: Orissa, Jammu and Kashmir, North eastern territorial states that due to various geographical and political barriers represent high inadequacy in dental health 
sector. On the other hand, in the states like Maharashtra, Punjab and Haryana, dentists are facing crisis to find jobs and struggle to establish due to ever-growing saturations and competitions. The skewed distribution also put additional physical stress on dentists in overburdened areas and mental stress.

\section{A THOUGHT FOR IMPROVEMENT}

Amendments are needed at every front to ensure deliverance of quality care. Funds are needed to be raised in this respect especially to reach to rural areas. More manpower aiming at equal distribution of services needs to be recruited. Providing quality to masses by means of providing quality life to dentists. That becomes a moral responsibility of administration to deal with underemployment, the gravest of all problems, at this scenario. Opening of new government colleges and putting a check on distribution of private colleges is another area where government considerations are may work for betterment. The timely inspections of educational and infrastructural facilities being provided by colleges is essential, as the quality of dentists being produced by the institutions govern the quality of care delivered. The major problem being encountered is the rising number of oral cancers and other oral diseases numbers despite of all efforts put forth till date. The reason behind is failure to diagnose the oral ailments at an earlier stages. It demands special thought to introduce diagnostic tools and equip dental personnel to make prompt diagnosis (WHO, 2003) and carry out preventive regimes to deal with such rampant oral disasters. The quality of dental care is not only determined by the number of restorations carried out or extractions done, but incorporating the right lifestyle and habits to ensure good oral health is sole aim of dentist and number of healthy individuals are indicative of quality of dental practice. The primordial and primary levels of prevention are equally important as secondary and tertiary levels. A lot has been done and a lot is still needed to be done. To produce able dentists imbibing ethics, skill, armamentarium, funds, perseverance, knowledge and hospitability is the responsibility of dental associations and faculty members. It is also essential to maintain a routine regulatory check on dental clinics and hospitals, be it private or government aided. If properly synchronized strategies harboring all the needs of both the consumers and dental personnel are implemented the day is not far off when every dentist will be delivering a quality dental care and every individual will be a recipient of the same.

\section{REFERENCES}

1. Jain H, Agarwal A. Current scenario and crisis facing dental college graduates in India. J Clin Diagn Res 2012;6:1-4

2. Institute of Medicine (IOM). Crossing the Quality Chasm: A New Health System for the 21st Century. Washington, D.C: National Academy Press; 2001

3. Entwistle V., Firnigl D., Ryan M., Francis J. \& Kinghorn P. Which experiences of health care delivery matter to service users and why? A critical interpretive synthesis and conceptual map. J Health Serv Res Policy. 2012. 17, 70-8.

4. Mahal, A. S. \& Shah, N. Implications of the growth of dental education in India. J Dent Edu. 2006.70, 884-91.

5. Alkhenizan A, Shaw C. Impact of Accreditation on the Quality of Healthcare Services: a Systematic Review of the Literature. Annals of Saudi Medicine. 2011; 31(4):407-416. doi:10.4103/0256-4947.83204.

6. Sbaraini A, Carter SM, Evans RW, Blinkhorn A. Experiences of dental care: what do patients value? BMC Health Services Research. 2012;12:177.

7. Vivek V. Challenges to dental education in India. Health Sci 2012;1:1

8. Calnan M, Dickinson M, Manley G. The quality of general dental care: public and users' perceptions. Quality in Health Care: QHC. 1999;8(3):149-153.

9. Guay AH. Access to dental care: solving the problem for underserved populations. J Am Dent Assoc. 2004;135(11):1599-1605.

10. Westmoreland TM, Watson KR. Redeeming Hollow Promises: The Case for Mandatory Spending on Health Care for American Indians and Alaska Natives. American Journal of Public Health. 2006;96(4):600-605.

11. Demographics \& Current Scenario With Respect To Dentists, Dental Institutions \& Dental Practices In India. Ahuja NK. 2012. Indian journal of dental science, 5, 78-98.

12. Ahuja NK, Parmar R. Demographics \& Current Scenario with Respect to Dentists, Dental Institutions \& Dental Practices in India. Indian Journal of Dental Sciences 2011;3(2):8-11. 
Source of support: Nil, Conflict of interest: None declared

Cite this article as:

Mehta N, Wadhwa A, Chawla N. Quality in Dental Practice: A Review. Int Healthcare Res J 2018;2(2):27-32. doi: 10.26440/IHRJ/02_01/163

AUTHOR AFFILIATIONS:

1. Senior Resident, Unit of Public Health Dentistry, Oral Health Sciences Centre, PGIMER, Chandigarh

2. BDS, Private Dental Practitioner

3. MDS, Private Dental Practitioner

Corresponding Author:

Dr. Nishant Mehta

Senior Resident

Unit of Public Health Dentistry

Oral Health Sciences Centre

PGIMER, Chandigarh
For article enquiry/author contact details, e-mail at: manuscriptenquiry.ihrj@gmail.com 